九州大学学術情報リポジトリ

Kyushu University Institutional Repository

\title{
Paleoceanography of the middle Eocene Arctic 0 cean based on geochemical measurements of biogenic matter
}

Ogawa, Yusuke

Department of Earth and Planetary Sciences, Graduate School of Sciences, Kyushu University

Takahashi, Kozo

Department of Earth and Planetary Sciences, Graduate School of Sciences, Kyushu University

Yamanaka, Toshiro

Graduate School of Natural Science and Technology, Okayama University

https://doi.org/10.5109/11806

出版情報: 九州大学大学院理学研究院紀要 : Series D, Earth and planetary sciences. 32 (1)， pp.31-48, 2008-02-01. Faculty of Science, Kyushu University

バージョン:

権利関係 : 
Mem. Fac. Sci., Kyushu Univ., Ser. D, Earth \& Planet. Sci., Vol. XXXII, No. 1, pp. 31-48, February 1, 2008

\title{
Paleoceanography of the middle Eocene Arctic Ocean based on geochemical measurements of biogenic matter
}

\author{
Yusuke Ogawa*, Kozo Takahashi ${ }^{*}$ and Toshiro Yamanaka ${ }^{* *}$
}

\begin{abstract}
Geochemical analyses of biogenic opal, total organic carbon (TOC), total sulfur (TS), and stable sulfur isotopic composition were conducted on the middle Eocene section of the ACEX cores obtained by IODP Expedition 302. The analyzed \%TS contents were high in all the examined intervals, indicating sufficient sea water was present in the deep layer of the paleo Arctic basin in contrast with the low salinity surface waters determined by freshwater siliceous microfossils. From the high \%TS in Lithological Unit 1/6, the extent of sea water supply from outside of the Arctic basin appeared to have increased after $45 \mathrm{Ma}$. The euxinic condition of the bottom water is suggested by the TOC-TS diagram. The anoxic environment was brought about by the estuarine type circulation pattern and salinity stratification. The light sulfur isotope composition $\left(\delta^{34} \mathrm{~S}\right)$ indicates the microbial sulfate reduction in an open system. The trend of $\delta^{34} \mathrm{~S}$ shift toward lower values with time is an indication of the history that the water exchange between the Arctic Ocean and the outside basin became progressively extensive. The high \% TS, the plotted pattern of TOC vs TS, and the extremely low sulfur isotopic values are unique and not comparable with the modern analogous such as the Black Sea. These results illustrate that the middle Eocene Arctic Ocean retained characteristically unique environments on the geochemical aspect.
\end{abstract}

Keywords: IODP Expedition 302, ACEX, biogenic opal, organic carbon, sulfur, pyrite, $\delta^{34} \mathrm{~S}$, euxinic condition

\section{Introduction}

The Arctic Ocean is only the region in the northern hemisphere today where perennial sea-ice is present. This region is important for the global climatic change because of the presence of the sea-ice, which reflects most of the sunlight and leads to the formation of cold dense water which in turn influences the global abyssal circulation (Holland et al., 2001). Despite of its importance, the history of the Arctic Ocean had not sufficiently been studied as continuous sediment record from the Arctic region was not available in the past (e.g., Kitchell and Clark, 1982; Burkry, 1984; and Dell'agnese and Clark, 1994). IODP Expedition 302, Arctic Coring Expedition (ACEX), recovered sediment cores on the

Manuscript received on 19 November 2007; accepted on 20 December 2007

* Department of Earth and Planetary Sciences, Graduate School of Sciences, Kyushu University, Hakozaki6-10-1, Fukuoka, 812-8581, JAPAN; kozo@geo.kyushu-u.ac.jp

** Graduate School of Natural Science and Technology, Okayama University 1-1, Naka 3-chome, Tsushima, Okayama, 700-8530, JAPAN 
Lomonosov Ridge in the central Arctic (Fig. 1) focusing on the reconstruction of the paleoenvironmental evolution of the Arctic Ocean. The early Eocene Epoch represents the warmest interval of the whole Cenozoic Era (Zachos et al., 2001). During this time interval paleogeography of the Arctic was different from the modern era. The connection between the Arctic and the world oceans was limited, and thus the Arctic Ocean was isolated (e.g., Akhmetiev and Beniamovsk, 2004). The Lomonosov Ridge where the drilling was conducted broke away from the Eurasian continental margin about $57 \mathrm{Ma}$ (e.g., Vogt et al., 1979). Since then the Lomonosov Ridge moved away and subsided as the seafloor spreading proceeded along the Gakkel Ridge.

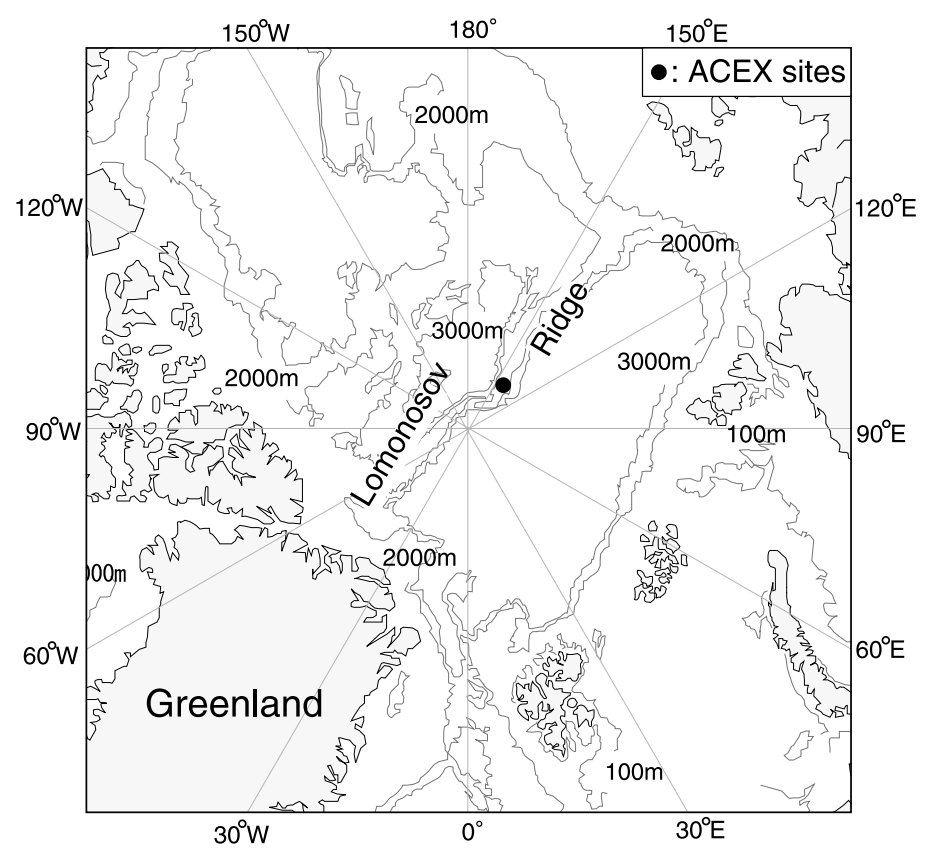

Fig. 1. Geographic map of the Arctic Ocean showing the locations of IODP Expedition 302 ACEX drill sites (solid circle) on the Lomonosov Ridge.

The early to middle Eocene section of the ACEX cores is characterized by abundant siliceous microfossils and organic carbon rich sediments. Siliceous microfossils are preserved in the sediments and referred to as biogenic opal. The biogenic opal content of marine sediments has been used to reconstruct past biological productivity in the surface ocean. This is based on the correspondence between modern sedimentary biogenic opal content and the productivity of surface waters (e.g., Leinen et al., 1986). Organic carbon in deep sea sediments is derived mainly from photosynthesis by marine phytoplankton. Therefore, the content of total organic carbon (TOC) also represents past productivity of surface waters analogous to biogenic opal (e.g., Müller and Suess, 1979).

The previous study on the microfossils indicated the low salinity environment in the Arctic Ocean during this period (Backman et al., 2006). Thus, the analyses of total sulfur (TS) content and stable sulfur isotopic composition are also of extreme interest because that they can provide important information whether the water masses were marine or freshwaters. Sulfur in reduced marine sediments 
is primarily contained as sulfides, especially pyrite (Berner, 1970). In anaerobic environment such as anoxic sediments and/or anoxic water column, $\mathrm{H}_{2} \mathrm{~S}$ is generated by sulfate-reducing bacteria, which reduce sea water sulfate ion using organic matter as an electron donor. The $\mathrm{H}_{2} \mathrm{~S}$ reacts readily with detrital iron minerals, resulting pyrite formation (Berner and Raiswell, 1983). Such sulfate reduction followed by pyrite formation has been commonly observed in the marine sediment. The constraints on pyrite formation are availability of sulfate ion $\left(\mathrm{SO}_{4}{ }^{2-}\right)$, reactive $\mathrm{Fe}$, and organic matter in addition to the extent of reducing condition. Accordingly, TS content can be used as marine water or freshwater proxy as the sulfate concentration of sea water is about $2700 \mathrm{ppm}$ in contrast with freshwater containing a few ppm of sulfate (e.g., Nakai et al., 1982). Another possible source of sedimentary sulfides is submarine volcanisms and hydrothermal activities. These sulfur types have markedly different sulfur isotopic compositions, i.e., bacterial sulfides have lower $\delta^{34} \mathrm{~S}$ values which are generally less than $-20 \%$, while volcanic or hydrothermally-derived sulfides are about 0 to $+5 \%$ (e.g., Ohmoto and Rye, 1979). The reduction of sea water sulfate by sulfate-reducing bacteria is the first step in the course of pyrite formation. This process occurs only under anoxic conditions, utilizing organic matter as energy source. Bacterial dissimilatory sulfate reduction is well known to accompany large fractionation of stable sulfur isotopes, i.e., leading an enrichment of ${ }^{32} \mathrm{~S}$ in hydrogen sulfide (e.g., Kaplan and Rittenberg, 1964). Laboratory cultivation experiment confirmed about 10 to $30 \%$ isotopic fractionation during sulfate reduction (Chambers and Trudinger, 1979). Most of the generated sulfides are oxidized through both biological and chemical process (Jørgensen, 1974). This oxidative part forms thiosulfate as an intermediate product (Moses et al., 1987). The thiosulfate can experience various transformations, i.e., oxidation to sulfate, reduction to sulfide, and disproportionation into sulfate and sulfide (Bak and Cypionka, 1987). The generated thiosulfate also has low sulfur isotopic value, and thus the rapid rereduction of the intermediate will also contribute extra isotopic fractionation of sulfur (Kajiwara, 1989). Many sulfate reducing bacteria are known to have the ability of disproportionation (Krämer and Cypionka, 1989). When bacteria disproportionate thiosulfate, ${ }^{34} \mathrm{~S}$ is enriched in sulfate, leading additional depletion of ${ }^{34} \mathrm{~S}$ in sulfide (Jørgensen, 1990). This process is thought to be important to explain the low sulfur isotopic value of sulfide in the marine sediments which cannot be achieved by the bacterial sulfate reduction alone (Jørgensen, 1990). On the other hand, no significant isotopic fractionation occurs during the pyrite formation from hydrogen sulfide (Böttcher et al., 1998). The isotopically low hydrogen sulfide is reacted with Fe ion and eventually preserved as sedimentary pyrite (Berner, 1984). During the pyrite formation, however, availability of sulfate ion affect strongly resulting sulfide $\delta^{34} \mathrm{~S}$ value, i.e., low sulfate concentration should limit the isotope discrimination. Therefore, stable isotope composition of pyrite in the sediments reflects the source of reduced sulfur and its microbial pathway.

Based on these proxies, in this study we focus on the reconstruction of the changes in paleoenvironmental conditions such as productivity, presence of sea water, and sulfur cycle of the middle Eocene Arctic Ocean. Biogenic opal content of the sediment was analyzed on 372 samples. The content of TOC, total nitrogen (TN), and TS were measured on 147 samples. Stable sulfur isotope of pyrite was also analyzed on 18 samples.

\section{Materials and Methods}

Integrated Ocean Drilling Program (IODP) Expedition 302, Arctic Coring Expedition (ACEX), recovered sediment cores on the Lomonosov Ridge of the central Arctic Ocean in 2004 (Fig. 1, Table 1). The cored sites are M0001, M0002, M0003, and M0004. The primary scientific objectives of Expedition 302 were to obtain the first continuous sediment records and to sample the underlying basement rock. The drilled depth reached $428 \mathrm{~m}$ thick sediments with the recovery of $68.4 \%$ and the 
ages ranged from the Quaternary at the top to the Late Cretaceous basement at the bottom (Backman et al., 2006). The sediment samples studied in this paper are the late early to the middle Eocene biosiliceous interval of the ACEX cores. The ages were determined by the biostratigraphic datums of 44.6 Ma, 48.6 Ma, and 55.0 Ma (Fig. 2: Backman et al., 2006). The lithology of the studied intervals is divided into Unit 1/6, Unit 2, and Unit 3; Unit 1/6, ranging from 198.13-220.24 meters below seafloor (mbsf), representing very dark gray, firm, homogenous silty clay to clayey silt; Unit 2, 220.24-313.61 mbsf, representing very dark gray mud-bearing siliceous ooze; and Unit 3, 313.61404.79 mbsf, representing clay and silty clay (Fig. 2: Backman et al., 2006). The top of Unit 3 comprises gray to very dark gray and firm to very firm clay. Two centimeter thick sedimentary samples were obtained at the interval of every $20 \mathrm{~cm}$, which were subjected to the analyses proceeded herein.

Table 1. Coring summary of Holes M0002A and M0004A in IODP Expedition 302 Arctic Coring Expedition (Backman et al., 2006).

\begin{tabular}{lcc}
\hline Hole & M0002A & M0004A \\
\hline Latitude & $87^{\circ} 55.271^{\prime} \mathrm{N}$ & $87^{\circ} 51.995^{\prime} \mathrm{N}$ \\
Longtitude & $139^{\circ} 21.901^{\prime} \mathrm{E}$ & $136^{\circ} 10.641^{\prime} \mathrm{E}$ \\
Water depth (sonar, m) & 1209 & 1287.9 \\
Total cored length $(\mathrm{m})$ & 270.1 & 157.59 \\
Total core recovered $(\mathrm{m})$ & 213.15 & 78.41 \\
Core recovery $(\%)$ & 78.9 & 49.8 \\
Bottom depth (mbsf) & 270.1 & 427.9 \\
Bottom age & middle Eocene & late Cretaceous \\
\hline
\end{tabular}

Prior to the chemical analyses the sediment samples were freeze-dried for 24 hours, followed by the procedure grinding into powder. The weight percent of biogenic opal was determined by following the procedures modified from Mortlock and Froelich (1989). Approximately $10 \mathrm{mg}$ of dried samples were weighed and treated with $10 \%$ hydrogen peroxide solution and $10 \% \mathrm{HCl}$ solution in order to remove organic matter and calcium carbonate. After washing the oxidant and acid, sample tubes were placed in an oven at $50^{\circ} \mathrm{C}$ for 24 hours for drying. Biogenic opal was extracted by $2-\mathrm{M} \mathrm{NaOH}$ solution at $85^{\circ} \mathrm{C}$ for 6 hours. Biogenic opal content was determined by molybdate-yellow spectrometry using Shimadzu UV mini-1240 Spectrophotometer.

For the analyses of total carbon (TC), TN, and TS, approximately $2 \mathrm{mg}$ of each dried sample was measured in a tin capsule. The determination was performed using Perkin-Elmer ${ }^{\circledR} 2400$ II CHNS/O Elementary Analyzer. Inorganic carbon content was found to be nearly zero in all intervals through the preliminary analyses, and thus TC was regarded as TOC content.

Sulfur isotopic compositions were measured using a continuous flow-isotope ratio mass spectrometer (IsoPrime ${ }^{\mathrm{TM}}$ EA, GV Instruments, UK) established at the Okayama University and were reported in the conventional $\delta^{34} \mathrm{~S}$ notation relative to Vienna Cañyon Diablo Troilite. The pulverized and dried sediment samples were converted into sulfate by heating with hot hydrogen peroxide solution. The resulting sulfates were recovered as $\mathrm{BaSO}_{4}$ by adding $\mathrm{BaCl}_{2}$ solution. The sulfate precipitations were wrapped in tin capsules for combustion in the elemental analyzer of the mass spectrometer. The overall reproducibility of the sulfur isotopic analysis was $\pm 0.2 \%$. 


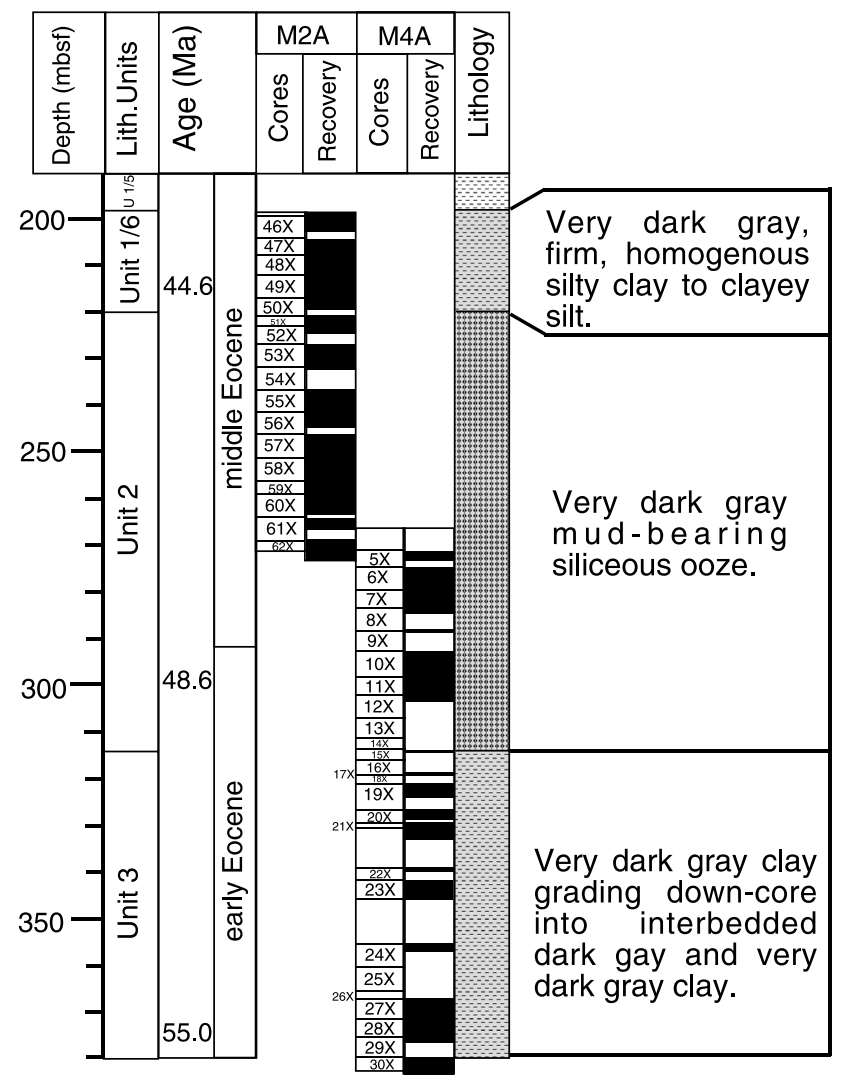

Fig. 2. Recovery and lithology of the sediments for Sites M0002A and M0004A. The black intervals represent recovered cores and the blank intervals represent no recovery (modified from Backman et al., 2006; the Azolla event from Brinkhuis et al., 2006).

Furthermore, in order to remove dilution effect, accumulation rates (AR; $\mathrm{g} \mathrm{cm}^{-2} \mathrm{kyr}^{-1}$ ) of biogenic opal, TOC, and TS were calculated by following equations

Biogenic opal AR $=\%$ Biogenic opal $*$ Dry bulk density $\left(\mathrm{g} \mathrm{cm}^{-3}\right) *$ Sedimentation rate $\left(\mathrm{cm} \mathrm{kyr}^{-1}\right) / 100$

TOC AR $=\% \mathrm{TOC}^{*}$ Dry bulk density*Sedimentation rate/100

TS AR $=\%$ TS* Dry bulk density*Sedimentation rate $/ 100$

\section{Results}

The determined values for \%biogenic opal, \%TOC, and \%TS and sulfur isotope composition are given in Table. 2 and Fig. 3. In addition, biogenic opal AR, TOC AR, and TS AR are shown in Fig. 4. The results and discussions in this study are presented by describing from the older sediments upward to the younger sediments as a standard format. 
Table 2. Geochemical analysed data for the Units 1/6, 2, and upper part of Unit 3.

\begin{tabular}{|c|c|c|c|c|c|c|c|c|c|c|c|c|c|c|c|c|c|c|c|c|c|c|}
\hline ¿ั & $\begin{array}{l}\text { 흘 } \\
\text { 을 } \\
\text { ஸे }\end{array}$ & 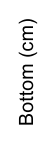 & $\begin{array}{l}\widehat{\underline{\xi}} \\
\frac{4}{\tilde{D}} \\
\frac{\Omega}{E}\end{array}$ & $\begin{array}{l}\bar{\pi} \\
\frac{0}{0} \\
.0 \\
\frac{0}{0} \\
\frac{0}{0} \\
\frac{0}{3} \\
.0 \\
\frac{0}{0}\end{array}$ & 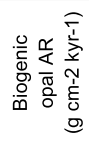 & 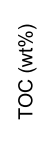 & $\frac{\widehat{o}}{\frac{0}{3}}$ & $\begin{array}{l}\widehat{o} \\
\stackrel{o}{\xi} \\
z\end{array}$ & $\begin{array}{l}\widehat{\widehat{े}} \\
\text { co } \\
\text { d }\end{array}$ & $\frac{z}{U}$ & ๖ัँ & 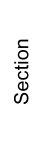 & $\begin{array}{l}\text { है을 } \\
\text { 음 }\end{array}$ & 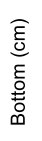 & $\begin{array}{l}\widehat{E} \\
\text { E્⿹ } \\
\text { है }\end{array}$ & 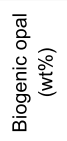 & 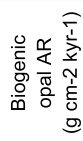 & 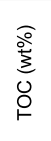 & $\begin{array}{l}\widehat{a} \\
\frac{0}{3} \\
0 \\
1\end{array}$ & $\begin{array}{l}\widehat{o} \\
\stackrel{a}{3} \\
z\end{array}$ & $\begin{array}{l}\widehat{0} \\
\text { के } \\
0\end{array}$ & $\underset{U}{z}$ \\
\hline \multicolumn{11}{|c|}{ Hole M0002A } & 48 & 2 & 96 & 98 & 208 & 10.3 & 0.34 & 2.0 & 14.4 & 0.1 & & 19.4 \\
\hline 46 & 114 & 16 & 197 & 11.1 & & 3.6 & 1.6 & 0.3 & & 13.9 & 48 & 2 & 116 & 118 & 208 & 11.4 & 0.35 & & & & & \\
\hline 46 & 156 & 58 & 198 & 8.4 & & 2.3 & 4.5 & 0.2 & & 14.2 & 48 & 2 & 136 & 138 & 208 & 12.6 & 0.38 & 2.4 & 10.4 & 0.2 & -55.0 & 18.8 \\
\hline 46 & 176 & 78 & 198 & 8.3 & & 5.3 & 4.1 & 0.3 & & 24.6 & 48 & 3 & 14 & 16 & 209 & 13.0 & 0.35 & & & & & \\
\hline 46 & 196 & 98 & 198 & 9.1 & & 1.6 & 2.4 & 0.1 & & 14.6 & 48 & 3 & 36 & 38 & 209 & 13.7 & 0.38 & 2.3 & 11.7 & 0.2 & & 11.7 \\
\hline 46 & 1116 & 118 & 198 & 5.8 & 0.18 & 3.7 & 21.2 & 0.2 & & 25.4 & 48 & 3 & 56 & 58 & 209 & 13.7 & 0.38 & & & & & \\
\hline 46 & 214 & 16 & 199 & 9.1 & 0.29 & & & & & & 48 & 3 & 76 & 78 & 209 & 14.3 & 0.42 & & & & & \\
\hline 46 & 234 & 36 & 199 & 10.2 & 0.33 & & & & & & 48 & 3 & 96 & 98 & 209 & 13.8 & 0.40 & & & & & \\
\hline 46 & 256 & 58 & 199 & 11.7 & 0.35 & 4.1 & 14.2 & 0.2 & -58.5 & 21.8 & 48 & 3 & 116 & 118 & 210 & 13.8 & 0.38 & 3.1 & 10.5 & 0.2 & & 17.9 \\
\hline 46 & 276 & 78 & 199 & 13.2 & 0.40 & & & & & & 48 & 3 & 136 & 138 & 210 & 14.2 & 0.40 & & & & & \\
\hline 46 & 296 & 98 & 199 & 12.2 & 0.38 & & & & & & 48 & 4 & 14 & 16 & 210 & 18.6 & 0.45 & & & & & \\
\hline 46 & 2116 & 118 & 200 & 12.8 & 0.42 & & & & & & 48 & 4 & 42 & 43 & 210 & 17.3 & 0.43 & & & & & \\
\hline 46 & 2136 & 138 & 200 & 16.7 & 0.52 & 2.8 & 12.0 & 0.2 & & 17.1 & 49 & 2 & 14 & 16 & 210 & & & 3.0 & 10.5 & 0.2 & & 19.6 \\
\hline 46 & $\begin{array}{ll}3 & 14\end{array}$ & 16 & 200 & 15.5 & 0.46 & & & & & & 49 & 2 & 37 & 39 & 211 & 15.6 & 0.41 & & & & & \\
\hline 46 & 334 & 36 & 200 & 16.5 & 0.49 & & & & & & 49 & 2 & 56 & 58 & 211 & 14.7 & 0.37 & & & & & \\
\hline 47 & $\begin{array}{ll}1 & 16\end{array}$ & 18 & 202 & 19.8 & 0.63 & & & & & & 49 & 2 & 76 & 78 & 211 & 16.2 & 0.44 & & & & & \\
\hline 47 & 214 & 16 & 202 & 22.2 & 0.69 & 2.8 & 9.0 & 0.2 & & 18.1 & 49 & 2 & 96 & 98 & 211 & 13.6 & 0.35 & 2.6 & 11.5 & 0.2 & & 18.8 \\
\hline 47 & 236 & 38 & 203 & 17.1 & 0.54 & & & & & & 49 & 2 & 116 & 118 & 211 & 12.1 & 0.34 & & & & & \\
\hline 47 & 256 & 58 & 203 & 15.0 & 0.39 & 4.0 & 13.6 & 0.3 & & 15.7 & 49 & 2 & 136 & 138 & 212 & 15.8 & 0.40 & & & & & \\
\hline 47 & 276 & 78 & 203 & 18.6 & 0.40 & 3.6 & 9.3 & 0.2 & & 19.1 & 49 & 3 & 14 & 16 & 212 & 14.6 & 0.38 & & & & & \\
\hline 47 & 296 & 98 & 203 & 37.4 & 0.56 & 3.4 & 9.5 & 0.3 & & 14.2 & 49 & 3 & 36 & 38 & 212 & 14.3 & 0.36 & 3.0 & 9.9 & 0.2 & & 23.4 \\
\hline 47 & 2116 & 118 & 203 & 30.4 & 0.45 & 4.4 & 10.3 & 0.3 & & 19.8 & 49 & 3 & 56 & 58 & 212 & 17.6 & 0.44 & & & & & \\
\hline 47 & $\begin{array}{ll}3 & 14\end{array}$ & 16 & 204 & 14.6 & 0.40 & 2.9 & 13.1 & 0.2 & & 18.0 & 49 & 3 & 76 & 78 & 212 & 13.9 & 0.39 & 2.3 & 10.5 & 0.3 & & 8.7 \\
\hline 47 & 336 & 38 & 204 & 21.5 & 0.50 & & & & & & 49 & 3 & 96 & 98 & 213 & 11.6 & 0.28 & & & & & \\
\hline 47 & 356 & 58 & 204 & 15.4 & 0.44 & 2.9 & 14.9 & 0.2 & & 19.8 & 49 & 3 & 116 & 118 & 213 & 14.8 & 0.37 & 3.2 & 10.6 & 0.1 & & 27.0 \\
\hline 47 & 376 & 78 & 205 & 14.6 & 0.41 & & & & & & 49 & 3 & 136 & 138 & 213 & 14.8 & 0.39 & & & & & \\
\hline 47 & 396 & 98 & 205 & 9.6 & 0.30 & & & & & & 49 & 4 & 14 & 16 & 213 & 15.2 & 0.40 & 2.4 & 9.5 & 0.2 & & 15.5 \\
\hline 47 & 3116 & 118 & 205 & 10.9 & 0.31 & 3.3 & 15.3 & 0.3 & & 14.6 & 49 & 4 & 36 & 38 & 214 & 13.6 & 0.39 & & & & & \\
\hline 47 & 3136 & 138 & 205 & 12.6 & 0.35 & & & & & & 49 & 4 & 56 & 58 & 214 & 14.1 & 0.38 & 2.7 & 9.0 & 0.2 & & 14.9 \\
\hline 47 & $\begin{array}{ll}4 & 14\end{array}$ & 16 & 205 & 12.8 & 0.38 & 2.9 & 15.4 & 0.3 & & 10.2 & 49 & 4 & 76 & 78 & 214 & 13.6 & 0.36 & & & & & \\
\hline 47 & $4 \quad 36$ & 38 & 206 & 10.3 & 0.32 & & & & & & 49 & 4 & 96 & 98 & 214 & 19.4 & 0.46 & 3.2 & 5.5 & 0.2 & & 23.6 \\
\hline 47 & 456 & 58 & 206 & 10.6 & 0.34 & 2.8 & 16.0 & 0.2 & & 19.1 & 49 & 4 & 116 & 118 & 214 & 18.8 & 0.45 & & & & & \\
\hline 47 & $\begin{array}{ll}476 \\
\end{array}$ & 78 & 206 & 9.6 & 0.32 & & & & & & 49 & 4 & 136 & 138 & 215 & 18.2 & 0.43 & 3.5 & 9.3 & 0.2 & & 21.2 \\
\hline 47 & 496 & 98 & 206 & 4.0 & 0.14 & 2.4 & 18.9 & 0.2 & & 14.4 & 49 & 5 & 14 & 16 & 215 & 16.8 & 0.39 & & & & & \\
\hline 47 & 4116 & 118 & 206 & 9.0 & 0.32 & & & & & & 49 & 5 & 56 & 58 & 215 & 14.6 & 0.42 & & & & & \\
\hline 47 & 4136 & 138 & 207 & 7.1 & 0.25 & 2.4 & 19.4 & 0.1 & & 19.9 & 50 & 1 & 14 & 16 & 215 & 16.7 & & 2.7 & 7.3 & 0.2 & & 21.0 \\
\hline 47 & $\begin{array}{ll}5 & 14\end{array}$ & 16 & 207 & 9.1 & 0.31 & & & & & & 50 & 1 & 36 & 38 & 215 & 11.8 & 0.30 & & & & & \\
\hline 47 & 536 & 38 & 207 & 7.6 & 0.28 & 2.2 & 15.6 & 0.2 & & 16.7 & 50 & 1 & 56 & 58 & 216 & 12.1 & 0.35 & 2.9 & 12.0 & 0.1 & & 25.9 \\
\hline 48 & 114 & 16 & 206 & 12.0 & & & & & & & 50 & 1 & 76 & 78 & 216 & 15.6 & 0.42 & & & & & \\
\hline 48 & 136 & 38 & 206 & 10.3 & 0.33 & 3.0 & 18.7 & 0.2 & & 21.5 & 50 & 1 & 96 & 98 & 216 & 17.6 & 0.40 & 3.4 & 4.0 & 0.2 & & 18.9 \\
\hline 48 & 156 & 58 & 206 & 12.5 & 0.37 & & & & & & 50 & 1 & 116 & 118 & 216 & 12.5 & 0.32 & & & & & \\
\hline 48 & 176 & 78 & 206 & 12.6 & 0.39 & 2.5 & 17.5 & 0.1 & -40.3 & 35.9 & 50 & 1 & 136 & 138 & 216 & 13.8 & 0.41 & 2.7 & 13.2 & 0.1 & & 22.1 \\
\hline 48 & 196 & 98 & 206 & 13.3 & & & & & & & 50 & 2 & 14 & 16 & 217 & 12.0 & 0.32 & & & & & \\
\hline 48 & 1116 & 118 & 207 & 11.8 & 0.37 & 3.3 & 16.9 & 0.2 & & 20.0 & 50 & 2 & 36 & 38 & 217 & 11.4 & 0.32 & 2.8 & 11.4 & 0.2 & -53.0 & 21.9 \\
\hline 48 & 1136 & 138 & 207 & 17.0 & 0.43 & & & & & & 50 & 2 & 56 & 58 & 217 & 11.8 & 0.35 & & & & & \\
\hline 48 & 214 & 16 & 207 & 11.0 & 0.36 & 2.2 & 15.7 & 0.1 & & 21.5 & 52 & 1 & 14 & 16 & 221 & 39.7 & 0.48 & 2.4 & 4.8 & 0.2 & & 14.2 \\
\hline 48 & 236 & 38 & 207 & 13.1 & 0.41 & & & & & & 52 & 1 & 36 & 38 & 221 & 42.0 & 0.59 & & & & & \\
\hline 48 & 256 & 58 & 208 & 9.4 & 0.31 & 1.9 & 14.4 & 0.1 & & 17.0 & 52 & 1 & 56 & 58 & 222 & 46.8 & 0.59 & 2.3 & 3.7 & 0.1 & & 19.0 \\
\hline 48 & 276 & 78 & 208 & 10.2 & 0.33 & & & & & & 52 & 1 & 76 & 78 & 222 & 47.8 & 0.63 & & & & & \\
\hline
\end{tabular}




\begin{tabular}{|c|c|c|c|c|c|c|c|c|c|c|c|c|c|c|c|c|c|c|c|c|c|c|}
\hline ঠั & 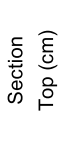 & 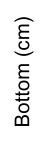 & 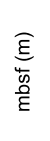 & $\begin{array}{l}\overline{0} \\
\text { 응 } \\
\frac{0}{0} \\
\frac{0}{0} \\
\frac{0}{0} \\
\frac{0}{0} \\
\frac{0}{0}\end{array}$ & 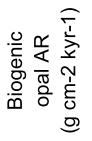 & 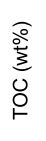 & $\frac{\widehat{o}}{\stackrel{2}{3}}$ & $\begin{array}{l}\text { ò } \\
\frac{+}{3} \\
z\end{array}$ & $\begin{array}{l}\text { ङ } \\
\text { की } \\
\text { के }\end{array}$ & $\underset{U}{z}$ & ঠั & 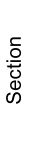 & $\begin{array}{l}\frac{\widehat{E}}{0} \\
\text { 음 }\end{array}$ & 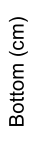 & $\begin{array}{l}\widehat{E} \\
\text { E⿱艹 } \\
\text { है }\end{array}$ & $\begin{array}{l}\overline{0} \\
\frac{0}{0} \\
.0 \\
\frac{0}{0} \\
\frac{0}{0} \\
\frac{0}{3} \\
\frac{0}{0}\end{array}$ & 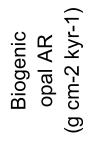 & 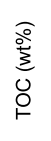 & 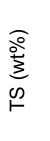 & $\begin{array}{l}\text { ò } \\
\stackrel{0}{3} \\
z \\
i=\end{array}$ & $\begin{array}{l}\text { बे } \\
\text { क } \\
\text { do }\end{array}$ & $\underset{U}{Z}$ \\
\hline 52 & 196 & 98 & 222 & 49.0 & 0.70 & 3.3 & 4.0 & 0.2 & & 18.3 & 55 & 4 & 136 & 138 & 239 & 44.0 & 0.71 & & & & & \\
\hline 52 & 1116 & 118 & 222 & 40.9 & 0.62 & & & & & & 55 & 5 & 14 & 16 & 240 & 45.4 & 0.65 & 2.4 & 3.7 & 0.1 & & 19.8 \\
\hline 52 & 1136 & 138 & 222 & 40.9 & 0.66 & 1.7 & 4.1 & 0.1 & & 16.4 & 55 & 5 & 36 & 38 & 240 & 42.6 & 0.68 & & & & & \\
\hline 53 & 114 & 16 & 225 & 48.7 & 0.55 & & & & & & 55 & 5 & 56 & 58 & 240 & 50.6 & 0.80 & 2.1 & 3.6 & 0.1 & & 41.6 \\
\hline 53 & 136 & 38 & 225 & 38.1 & 0.72 & 1.6 & 4.1 & 0.1 & & 17.2 & 56 & 1 & 14 & 16 & 240 & 58.4 & 0.70 & & & & & \\
\hline 53 & 156 & 58 & 226 & 45.2 & 0.73 & & & & & & 56 & 1 & 36 & 38 & 240 & 48.3 & & 2.6 & 3.7 & 0.2 & -44.9 & 17.9 \\
\hline 53 & 176 & 78 & 226 & 45.0 & 0.67 & 3.0 & & 0.2 & & 22.0 & 56 & 1 & 56 & 58 & 240 & 45.9 & 0.65 & & & & & \\
\hline 53 & 196 & 98 & 226 & 48.0 & 0.69 & & & & & & 56 & 2 & 14 & 16 & 240 & 55.2 & 0.69 & 2.9 & 3.5 & 0.1 & & 42.6 \\
\hline 53 & 1116 & 118 & 226 & 49.8 & 0.72 & 2.5 & 4.5 & 0.1 & & 22.4 & 56 & 2 & 36 & 38 & 241 & 47.6 & 0.68 & & & & & \\
\hline 53 & 1136 & 138 & 226 & 42.9 & 0.67 & & & & & & 56 & 2 & 56 & 58 & 241 & 49.9 & 0.73 & 2.6 & 3.6 & 0.2 & & 15.9 \\
\hline 53 & 214 & 16 & 227 & 57.6 & 0.68 & & & & & & 56 & 2 & 76 & 78 & 241 & 44.1 & 0.71 & & & & & \\
\hline 53 & 236 & 38 & 227 & 67.2 & & & & & & & 56 & 2 & 96 & 98 & 241 & 45.0 & 0.73 & 2.0 & 3.5 & 0.2 & & 13.7 \\
\hline 53 & 256 & 58 & 227 & 65.2 & 0.74 & 3.3 & 2.9 & 0.1 & & 29.3 & 56 & 2 & 116 & 118 & 241 & 54.3 & 0.74 & & & & & \\
\hline 53 & 276 & 78 & 227 & 46.0 & 0.73 & & & & & & 56 & 2 & 136 & 138 & 242 & 59.7 & 0.73 & 2.9 & 3.3 & 0.2 & & 18.5 \\
\hline 53 & 296 & 98 & 227 & 49.7 & 0.70 & 2.6 & 4.3 & 0.2 & & 13.9 & 56 & 3 & 14 & 16 & 242 & 57.3 & 0.70 & & & & & \\
\hline 53 & 2116 & 118 & 228 & 44.1 & 0.66 & & & & & & 56 & 3 & 36 & 38 & 242 & 56.0 & 0.84 & 2.4 & 3.5 & 0.2 & & 13.1 \\
\hline 53 & 2136 & 138 & 228 & 47.7 & 0.76 & & & & & & 56 & 3 & 56 & 58 & 242 & 53.0 & 0.73 & & & & & \\
\hline 53 & $\begin{array}{ll}3 & 14\end{array}$ & 16 & 228 & 51.3 & 0.69 & & & & & & 56 & 3 & 76 & 78 & 243 & 50.7 & 0.74 & & & & & \\
\hline 53 & 336 & 38 & 228 & 42.7 & 0.82 & 2.0 & 4.8 & 0.1 & & 25.7 & 56 & 3 & 96 & 98 & 243 & 64.0 & 0.76 & & & & & \\
\hline 53 & 356 & 58 & 229 & 40.0 & 0.67 & & & & & & 56 & 3 & 116 & 118 & 243 & 50.2 & 0.70 & 3.1 & 4.9 & 0.2 & & 16.2 \\
\hline 53 & 376 & 78 & 229 & 51.3 & 0.78 & & & & & & 57 & 2 & 14 & 16 & 245 & 52.6 & 0.66 & & & & & \\
\hline 53 & 396 & 98 & 229 & 54.7 & 0.68 & & & & & & 57 & 2 & 36 & 38 & 245 & 60.1 & 0.76 & & & & & \\
\hline 53 & 3116 & 118 & 229 & 58.0 & 0.71 & 3.3 & 3.6 & 0.3 & & 11.2 & 57 & 2 & 56 & 58 & 245 & 51.1 & 0.67 & & & & & \\
\hline 53 & 3136 & 138 & 229 & 51.4 & 0.70 & & & & & & 57 & 2 & 76 & 78 & 245 & 48.8 & 0.68 & 3.2 & 4.4 & 0.2 & & 16.4 \\
\hline 53 & $4 \quad 14$ & 16 & 230 & 54.5 & 0.71 & & & & & & 57 & 2 & 96 & 98 & 246 & 50.2 & 0.66 & & & & & \\
\hline 53 & 436 & 38 & 230 & 60.9 & 0.71 & & & & & & 57 & 2 & 116 & 118 & 246 & 56.6 & 0.65 & 2.7 & 3.8 & 0.2 & & 18.4 \\
\hline 53 & 456 & 58 & 230 & 56.3 & 0.70 & 3.5 & 4.1 & 0.2 & -34.9 & 17.2 & 57 & 2 & 136 & 138 & 246 & 58.5 & 0.69 & & & & & \\
\hline 54 & 114 & 16 & 230 & 50.3 & 0.64 & & & & & & 57 & 3 & 14 & 16 & 246 & 61.2 & 0.68 & & & & & \\
\hline 54 & 132 & 34 & 230 & 48.4 & 0.65 & & & & & & 57 & 3 & 36 & 38 & 247 & 43.1 & 0.60 & & & & & \\
\hline 55 & 214 & 16 & 235 & 49.1 & 0.66 & & & & & & 57 & 3 & 56 & 58 & 247 & 56.4 & 0.68 & 2.7 & 3.2 & 0.2 & & 16.8 \\
\hline 55 & 236 & 38 & 235 & 47.5 & 0.71 & 2.2 & 4.4 & 0.1 & -41.3 & 21.6 & 57 & 3 & 96 & 98 & 247 & 63.1 & 0.70 & & & & & \\
\hline 55 & 256 & 58 & 236 & 41.8 & 0.71 & & & & & & 57 & 3 & 116 & 118 & 247 & 65.1 & 0.75 & & & & & \\
\hline 55 & 276 & 78 & 236 & 49.8 & 0.72 & & & & & & 57 & 3 & 136 & 138 & 248 & 56.5 & 0.67 & 2.9 & 3.1 & 0.2 & & 18.5 \\
\hline 55 & 296 & 98 & 236 & 50.0 & 0.67 & & & & & & 57 & 4 & 14 & 16 & 248 & 55.4 & 0.64 & & & & & \\
\hline 55 & 2116 & 118 & 236 & 46.5 & 0.76 & 2.1 & 3.8 & 0.2 & & 11.4 & 57 & 4 & 36 & 38 & 248 & 65.5 & 0.76 & & & & & \\
\hline 55 & 2136 & 138 & 236 & 50.8 & 0.69 & & & & & & 57 & 4 & 56 & 58 & 248 & 61.1 & 0.69 & & & & & \\
\hline 55 & 314 & 16 & 237 & 44.1 & 0.69 & 2.2 & 3.8 & 0.2 & & 15.8 & 57 & 4 & 76 & 78 & 249 & 59.7 & 0.71 & & & & & \\
\hline 55 & 336 & 38 & 237 & 47.2 & 0.66 & & & & & & 57 & 4 & 96 & 98 & 249 & 59.4 & 0.70 & & & & & \\
\hline 55 & 356 & 58 & 237 & 42.4 & 0.71 & 2.2 & 4.5 & 0.1 & & 23.1 & 57 & 4 & 116 & 118 & 249 & 60.6 & 0.74 & 3.1 & 3.6 & 0.2 & & 16.2 \\
\hline 55 & 376 & 78 & 237 & 39.7 & 0.68 & & & & & & 57 & 4 & 136 & 138 & 249 & 53.6 & 0.75 & & & & & \\
\hline 55 & 396 & 98 & 238 & 47.9 & 0.71 & 2.0 & 4.0 & 0.2 & & 14.4 & 57 & 5 & 14 & 16 & 249 & 63.5 & 0.70 & & & & & \\
\hline 55 & 3116 & 118 & 238 & 50.9 & 0.72 & & & & & & 57 & 5 & 30 & 32 & 250 & 61.0 & 0.70 & & & & & \\
\hline 55 & 3136 & 138 & 238 & 51.4 & 0.72 & 2.6 & 4.6 & 0.1 & & 37.2 & 57 & 5 & 56 & 58 & 250 & 62.4 & 0.74 & 2.9 & 2.6 & 0.4 & & 8.6 \\
\hline 55 & $4 \quad 14$ & 16 & 238 & 52.9 & 0.74 & & & & & & 58 & 1 & 14 & 16 & 250 & 51.7 & & & & & & \\
\hline 55 & 436 & 38 & 238 & 47.0 & 0.71 & & & & & & 58 & 1 & 36 & 38 & 250 & 62.3 & 0.75 & & & & & \\
\hline 55 & 456 & 58 & 239 & 42.3 & 0.69 & & & & & & 58 & 1 & 56 & 58 & 250 & 59.1 & 0.70 & & & & & \\
\hline 55 & 476 & 78 & 239 & 62.9 & 0.68 & & & & & & 58 & 1 & 76 & 78 & 250 & 48.9 & 0.59 & 4.6 & 6.1 & 0.3 & -30.5 & 16.3 \\
\hline 55 & 496 & 98 & 239 & 37.5 & 0.69 & & & & & & 58 & 1 & 96 & 98 & 251 & 57.1 & 0.72 & & & & & \\
\hline 55 & 4116 & 118 & 239 & 37.2 & 0.73 & 1.8 & 5.6 & 0.3 & & 7.4 & 58 & 1 & 116 & 118 & 251 & 55.3 & 0.72 & & & & & \\
\hline
\end{tabular}


Table 2. (continued)

\begin{tabular}{|c|c|c|c|c|c|c|c|c|c|c|c|c|c|c|c|c|c|c|c|c|c|}
\hline 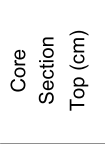 & $\begin{array}{l}\widehat{\bar{E}} \\
\bar{E} \\
\overline{0} \\
\overline{0} \\
0\end{array}$ & $\begin{array}{l}\widehat{\xi} \\
\text { हू" } \\
\text { है }\end{array}$ & 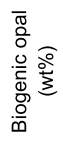 & 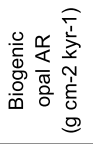 & 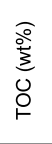 & 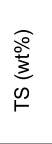 & 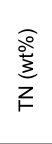 & $\begin{array}{l}\widehat{\text { o }} \\
\text { क } \\
\text { o } \\
0\end{array}$ & $z_{0}$ & ¿ัँ & & $\begin{array}{l}\widehat{\hat{c}} \\
\stackrel{0}{\circ} \\
\vdash\end{array}$ & 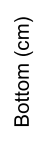 & $\begin{array}{l}\widehat{\underline{\xi}} \\
\text { हू } \\
\text { है }\end{array}$ & 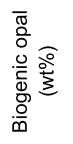 & 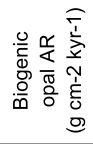 & $\begin{array}{l}\widehat{o} \\
\text { ò } \\
0 \\
0 \\
\vdash\end{array}$ & 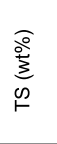 & $\begin{array}{l}\text { ò } \\
\text { 迹 } \\
z\end{array}$ & $\begin{array}{l}\text { बे } \\
\text { के } \\
0 \\
\text { के }\end{array}$ & z \\
\hline $58 \quad 1136$ & 1382 & 251 & 38.9 & 0.59 & & & & & & 61 & 2 & 14 & 16 & 264 & 54.5 & 0.73 & 2.8 & 4.6 & 0.2 & & 20.7 \\
\hline $\begin{array}{lll}58 & 2 & 14\end{array}$ & 162 & 251 & 48.6 & 0.66 & 2.9 & 4.5 & 0.2 & & 14.2 & 61 & 2 & 36 & 38 & 264 & 47.6 & 0.69 & 4.6 & 7.2 & 0.2 & & 23.8 \\
\hline $\begin{array}{lll}58 & 2 & 36\end{array}$ & 382 & 251 & 45.5 & 0.64 & & & & & & 61 & 2 & 56 & 58 & 265 & 46.5 & 0.72 & & & & & \\
\hline $\begin{array}{lll}58 & 2 \quad 56\end{array}$ & 582 & 252 & 53.3 & 0.67 & & & & & & 61 & 2 & 76 & 78 & 265 & 48.4 & 0.78 & & & & & \\
\hline $\begin{array}{lll}58 & 2 & 76\end{array}$ & 782 & 252 & 57.1 & 0.68 & & & & & & 61 & 2 & 96 & 98 & 265 & 52.3 & 0.77 & & & & & \\
\hline $\begin{array}{lll}58 & 2 & 96\end{array}$ & 982 & 252 & 65.5 & & 2.7 & 3.6 & 0.2 & & 17.8 & 61 & 2 & 116 & 118 & 265 & 56.8 & 0.62 & & & & & \\
\hline $58 \quad 2116$ & 1182 & 252 & 55.6 & 0.66 & & & & & & 62 & 1 & 96 & 98 & 269 & 51.7 & 0.68 & & & & & \\
\hline $58 \quad 2136$ & 1382 & 252 & 54.7 & 0.64 & & & & & & 62 & 1 & 116 & 118 & 269 & 52.1 & 0.75 & 3.2 & 4.9 & 0.2 & & 20.9 \\
\hline $\begin{array}{lll}58 & 3 & 14\end{array}$ & 162 & 253 & 60.8 & & & & & & & 62 & 1 & 136 & 138 & 269 & 48.6 & 0.66 & & & & & \\
\hline $\begin{array}{lll}58 & 3 & 36\end{array}$ & 38 & 253 & 56.2 & 0.65 & 3.9 & 4.3 & 0.2 & & 19.1 & 62 & 2 & 14 & 16 & 269 & 58.8 & & & & & & \\
\hline $\begin{array}{lll}58 & 3 & 56\end{array}$ & 58 & 253 & 53.7 & 0.64 & & & & & & 62 & 2 & 36 & 38 & 269 & 60.2 & 0.78 & & & & & \\
\hline $\begin{array}{lll}58 & 3 & 76\end{array}$ & 78 & 253 & 51.7 & 0.69 & & & & & & 62 & 2 & 56 & 58 & 270 & 50.7 & 0.73 & 3.0 & 5.2 & 0.2 & & 19.7 \\
\hline $\begin{array}{lll}58 & 3 & 96\end{array}$ & 98 & 254 & 50.9 & 0.62 & & & & & & 62 & 2 & 76 & 78 & 270 & 49.3 & 0.69 & & & & & \\
\hline $58 \quad 3116$ & 118 & 254 & 55.0 & 0.69 & 3.1 & 4.2 & 0.2 & & 18.8 & 62 & 2 & 96 & 98 & 270 & 56.3 & 0.75 & 3.1 & 4.9 & 0.2 & & 20.4 \\
\hline $58 \quad 3136$ & 138 & 254 & 51.8 & 0.64 & & & & & & 62 & 2 & 116 & 118 & 270 & 50.4 & 0.70 & & & & & \\
\hline $\begin{array}{lll}58 & 4 & 14\end{array}$ & 16 & 254 & 51.7 & 0.63 & & & & & & 62 & 2 & 136 & 138 & 270 & 53.8 & 0.73 & 4.4 & 6.3 & 0.3 & -39.6 & 16.6 \\
\hline $\begin{array}{lll}58 & 4 & 20\end{array}$ & 22 & 254 & 52.2 & 0.63 & & & & & & 62 & 3 & 14 & 16 & 271 & 51.8 & 0.71 & & & & & \\
\hline $58 \quad 4 \quad 56$ & 58 & 255 & 60.4 & & 3.4 & 3.7 & 0.2 & & 20.9 & 62 & 3 & 36 & 38 & 271 & 51.6 & 0.74 & & & & & \\
\hline 592116 & 118 & 257 & 59.7 & 0.66 & & & & & & 62 & 3 & 56 & 58 & 271 & 49.5 & 0.69 & & & & & \\
\hline 592136 & 138 & 257 & 62.6 & 0.71 & & & & & & 62 & 3 & 76 & 78 & 271 & 55.0 & 0.80 & 3.4 & 5.2 & 0.2 & & 19.7 \\
\hline $\begin{array}{lll}59 & 3 & 14\end{array}$ & 16 & 258 & 55.5 & 0.63 & & & & & & & & & & & & & & & & & \\
\hline $\begin{array}{lll}59 & 3 & 36\end{array}$ & 38 & 258 & 57.8 & 0.77 & 3.1 & 4.0 & 0.2 & & 22.3 & & le M & noo04 & & & & & & & & & \\
\hline $\begin{array}{lll}59 & 3 & 56\end{array}$ & 58 & 258 & 57.9 & 0.66 & & & & & & 6 & 1 & 14 & 16 & 274 & 53.7 & 0.60 & & & & & \\
\hline $\begin{array}{lll}59 & 3 & 76\end{array}$ & 78 & 258 & 63.3 & 0.73 & & & & & & 6 & 1 & 36 & 38 & 274 & 51.9 & 0.61 & & & & & \\
\hline $\begin{array}{lll}59 & 3 & 96\end{array}$ & 98 & 259 & 59.6 & 0.68 & & & & & & 6 & 1 & 56 & 58 & 274 & 53.8 & 0.68 & & & & & \\
\hline 593116 & 118 & 259 & 67.7 & 0.77 & 2.8 & 3.6 & 0.2 & & 17.0 & 6 & 1 & 76 & 78 & 274 & 47.7 & 0.61 & & & & & \\
\hline $\begin{array}{lll}60 & 1 & 14\end{array}$ & 16 & 258 & 54.3 & & & & & & & 6 & 1 & 96 & 98 & 274 & 52.6 & 0.65 & 3.0 & 5.3 & 0.2 & & 19.7 \\
\hline 60156 & 58 & 258 & 56.9 & 0.70 & & & & & & 6 & 1 & 116 & 118 & 275 & 48.9 & 0.59 & & & & & \\
\hline $\begin{array}{lll}60 & 1 & 76\end{array}$ & 78 & 258 & 62.7 & 0.79 & 2.9 & 3.8 & 0.2 & & 19.6 & 6 & 1 & 136 & 138 & 275 & 50.4 & 0.66 & & & & & \\
\hline $60 \quad 196$ & 98 & 259 & 55.0 & 0.74 & & & & & & 6 & 2 & 14 & 16 & 275 & 52.0 & 0.68 & & & & & \\
\hline $60 \quad 1116$ & 118 & 259 & 46.1 & 0.70 & & & & & & 6 & 2 & 36 & 38 & 275 & 49.2 & 0.65 & 2.7 & 5.0 & 0.2 & & 19.8 \\
\hline $60 \quad 1136$ & 138 & 259 & 49.5 & 0.80 & & & & & & 6 & 2 & 56 & 58 & 276 & 49.2 & 0.63 & & & & & \\
\hline $\begin{array}{lll}60 & 2 & 14\end{array}$ & 16 & 259 & & & 4.0 & 5.3 & 0.2 & & 23.2 & 6 & 2 & 76 & 78 & 276 & 50.8 & 0.67 & & & & & \\
\hline $\begin{array}{lll}60 & 2 & 36\end{array}$ & 38 & 259 & 53.3 & 0.74 & & & & & & 6 & 2 & 96 & 98 & 276 & 47.8 & 0.67 & & & & & \\
\hline $60 \quad 2 \quad 56$ & 58 & 260 & 57.0 & 0.89 & 3.5 & 5.2 & 0.2 & & 22.4 & 6 & 2 & 116 & 118 & 276 & 47.0 & 0.69 & 3.7 & 5.9 & 0.2 & -38.9 & 23.7 \\
\hline $\begin{array}{lll}60 & 2 & 76\end{array}$ & 78 & 260 & 51.3 & 0.78 & & & & & & 6 & 2 & 136 & 138 & 276 & 45.6 & & & & & & \\
\hline $60 \quad 296$ & 98 & 260 & 55.8 & 0.81 & 3.7 & 5.1 & 0.2 & & 21.5 & 6 & 3 & 14 & 16 & 277 & 52.5 & 0.69 & 3.4 & 5.1 & 0.2 & & 22.2 \\
\hline $60 \quad 2116$ & 118 & 260 & 55.4 & 0.86 & & & & & & 6 & 3 & 36 & 38 & 277 & 54.2 & 0.64 & & & & & \\
\hline $60 \quad 2136$ & 138 & 260 & 50.6 & 0.76 & & & & & & 6 & 3 & 56 & 58 & 277 & 53.0 & 0.71 & & & & & \\
\hline $\begin{array}{lll}60 & 3 & 36\end{array}$ & 38 & 261 & 55.1 & 0.81 & 2.3 & 3.7 & 0.1 & -38.5 & 20.6 & 6 & 3 & 76 & 78 & 277 & 49.9 & 0.61 & & & & & \\
\hline $\begin{array}{lll}60 & 3 & 56\end{array}$ & 58 & 261 & 52.2 & 0.69 & & & & & & 6 & 3 & 96 & 98 & 277 & 58.1 & 0.78 & 2.5 & 3.9 & 0.2 & & 16.3 \\
\hline $\begin{array}{lll}60 & 3 & 76\end{array}$ & 78 & 261 & 70.3 & & & & & & & 6 & 3 & 116 & 118 & 278 & 52.2 & 0.70 & & & & & \\
\hline $\begin{array}{lll}60 & 3 & 96\end{array}$ & 98 & 262 & 46.8 & 0.69 & & & & & & 6 & 3 & 136 & 138 & 278 & 64.8 & 0.70 & & & & & \\
\hline 61156 & 58 & 263 & 55.0 & 0.73 & 4.2 & 6.4 & 0.2 & & 20.8 & 6 & 4 & 14 & 16 & 278 & 54.4 & 0.61 & & & & & \\
\hline $\begin{array}{lll}61 & 1 & 76\end{array}$ & 78 & 263 & 50.6 & 0.74 & 3.2 & 5.0 & 0.2 & & 19.5 & 6 & 4 & 36 & 38 & 278 & 54.6 & 0.62 & 2.4 & 4.1 & 0.1 & & 25.5 \\
\hline 611996 & 98 & 264 & 53.5 & 0.73 & 3.3 & 5.2 & 0.2 & & 19.4 & 7 & 1 & 14 & 16 & 279 & 57.2 & 0.74 & & & & & \\
\hline 611116 & 118 & 264 & 51.3 & 0.75 & 3.9 & 5.7 & 0.2 & & 21.7 & 7 & 1 & 36 & 38 & 279 & 51.5 & 0.71 & & & & & \\
\hline $61 \quad 1136$ & 138 & 264 & 50.9 & 0.79 & 4.0 & 6.4 & 0.2 & & 24.6 & 7 & 1 & 56 & 58 & 279 & 64.3 & 0.78 & & & & & \\
\hline
\end{tabular}




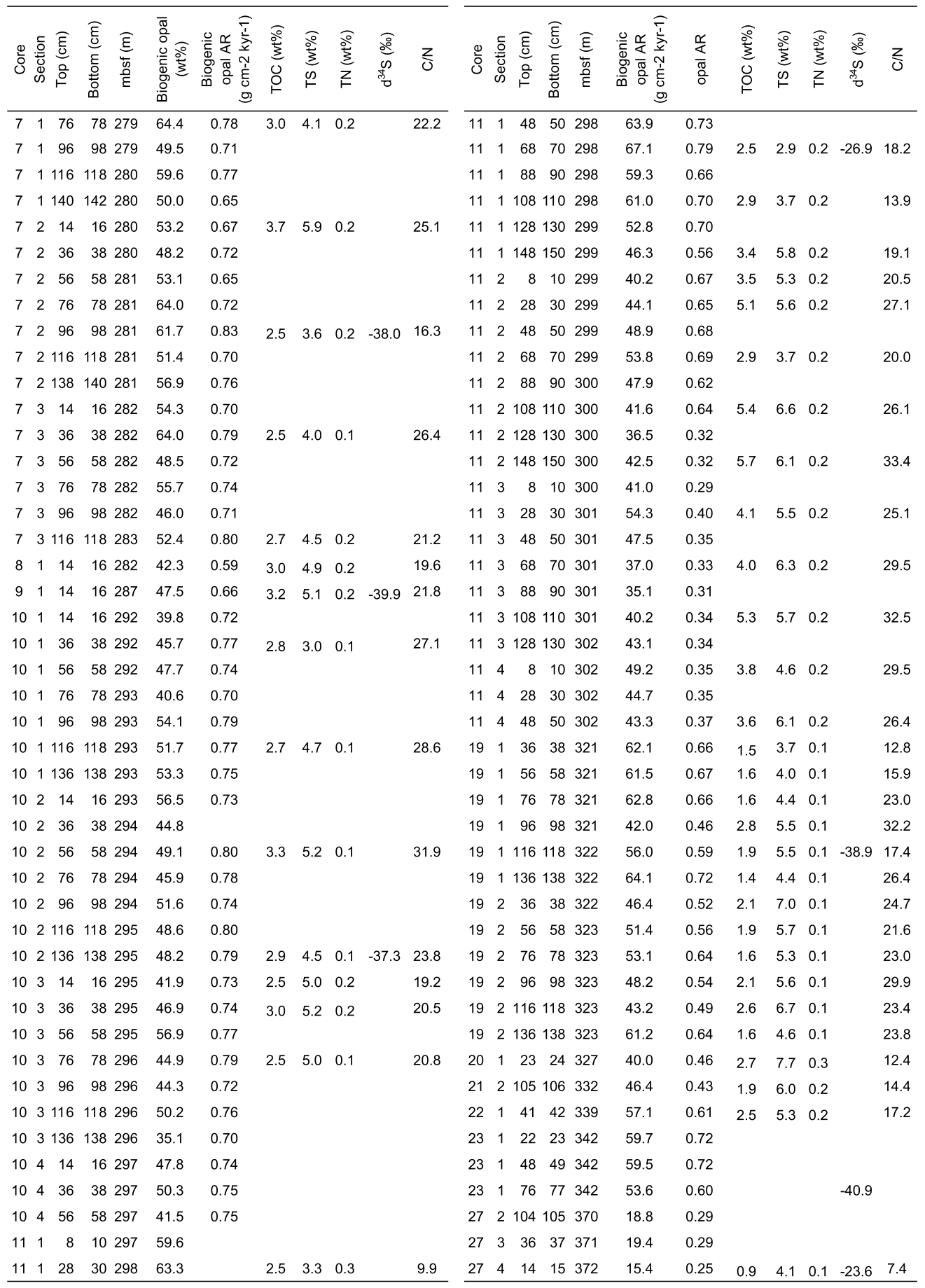




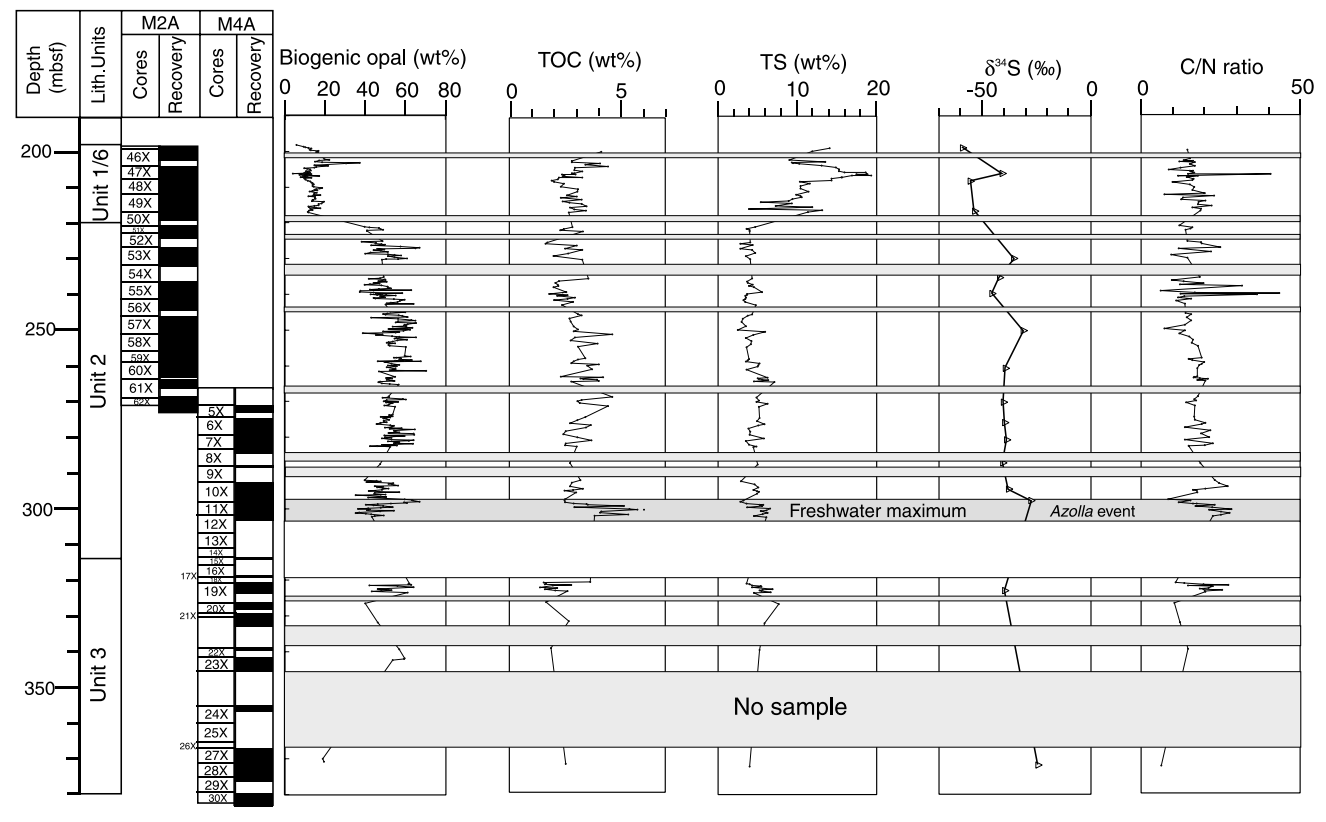

Fig. 3. Changes in biogenic opal (wt $\%$ ), TOC (wt $\%)$, TS (wt $\%), \delta^{34} \mathrm{~S}$, and $\mathrm{C} / \mathrm{N}$ ratio in the ACEX sediments (Lithology units and recovery from Backman et al., 2006).

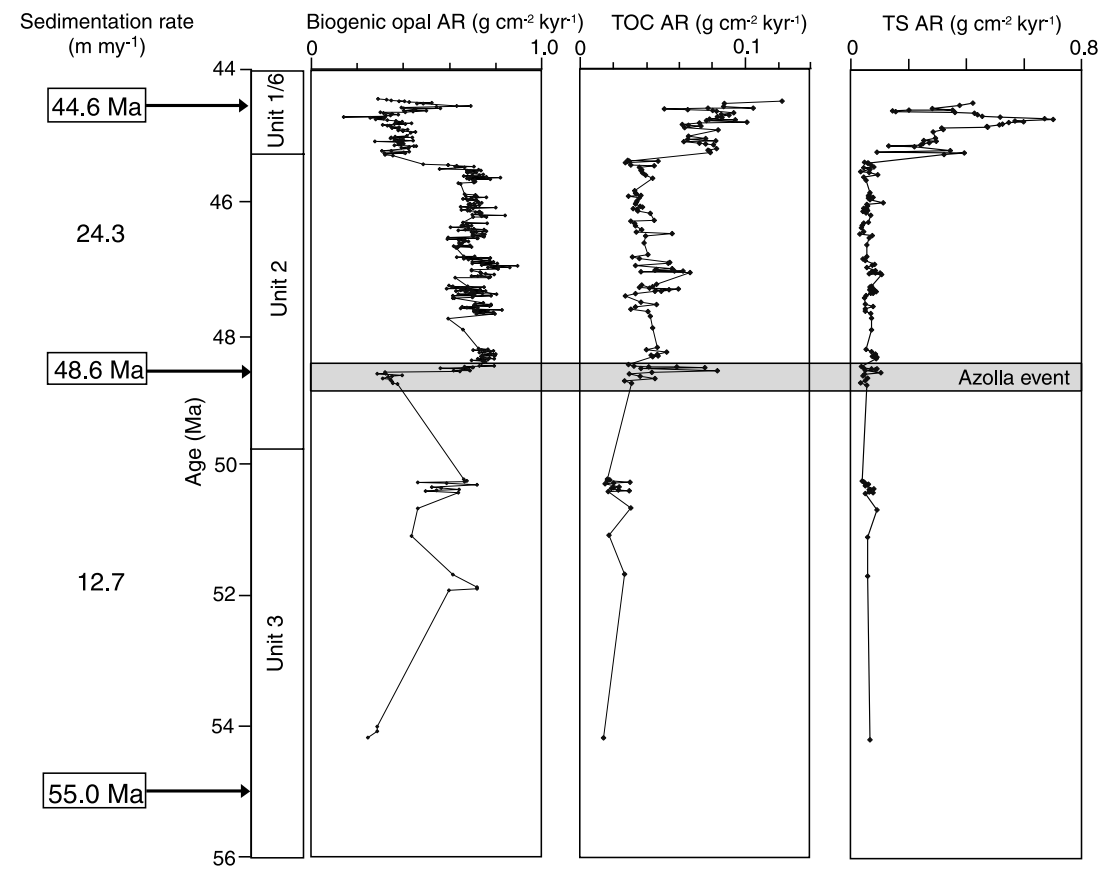

Fig. 4. Changes in accumulation rates of biogenic opal, TOC, and TS. 


\subsection{Biogenic opal and TOC}

The \%biogenic opal varied significantly especially with the lithological change between Units 2 and 1/6. In the upper part of Unit 3 and Unit 2, biogenic opal represented very high and it showed highamplitude variations. The \%biogenic opal ranged from 15.4 to $64.1 \%$ in Unit 3, and from 35.1 to $70.3 \%$ in Unit 2. The biogenic opal content decreased significantly in Unit 1/6 compared to those in Unit 2, generally ranging from 10 to $20 \%$, but full range spanned from 4.0 to $37.4 \%$. The amplitude in Unit $1 / 6$ was low. Biogenic opal AR ranged from 0.25 to $0.72 \mathrm{~g} \mathrm{~cm}^{-2} \mathrm{kyr}^{-1}$ in Unit 3, from 0.29 to $0.89 \mathrm{~g} \mathrm{~cm}^{-2} \mathrm{kyr}^{-1}$ in Unit 2, and from 0.14 to $0.69 \mathrm{~g} \mathrm{~cm}^{-2} \mathrm{kyr}^{-1}$ in Unit $1 / 6$.

In contrast with biogenic opal, there was no significant change in $\% \mathrm{TOC}$ between Unit 2 and $1 / 6$. The $\%$ TOC showed high values in all intervals with high amplitude. The \%TOC ranged from 0.9 to $2.8 \%$ in Unit 3, from 1.62 to $5.7 \%$ in Unit 2, and from 1.9 to $4.4 \%$ in Unit $1 / 6$. TOC AR ranged from 0.01 to $0.03 \mathrm{~g}$ $\mathrm{cm}^{-2} \mathrm{kyr}^{-1}$ in Unit 3, from 0.02 to $0.08 \mathrm{~g} \mathrm{~cm}^{-2} \mathrm{kyr}^{-1}$ in Unit 2, and from 0.05 to $0.13 \mathrm{~g} \mathrm{~cm}^{-2} \mathrm{kyr}^{-1}$ in Unit $1 / 6$.

\subsection{TS and Sulfur isotopic composition}

Sulfur is contained in the ACEX sediments mainly as pyrite, which occurred as framboidal structure based on the scanning electron microscopic observation (Fig. 5). The framboidal pyrite generally has been considered microbial in origin (Berner, 1970). The \%TS represented high values in all the intervals investigated. The \%TS markedly increased from Unit 2 to Unit $1 / 6$ at the expense of \%biogenic opal decrease. The \%TS ranged from 3.7 to $7.7 \%$ in Unit 3, from 2.6 to $7.2 \%$ in Unit 2, and from 4.0 to $19.4 \%$ in Unit 1/6. The TS AR ranged from 0.04 to $0.09 \mathrm{~g} \mathrm{~cm}^{-2} \mathrm{kyr}^{-1}$ in Unit 3, from 0.03 to $0.11 \mathrm{~g} \mathrm{~cm}^{-2} \mathrm{kyr}^{-1}$ in Unit 2, and from 0.09 to $0.73 \mathrm{~g} \mathrm{~cm}^{-2} \mathrm{kyr}^{-1}$ in Unit $1 / 6$. The TS AR was calculated for the comparison purpose with opal and organic carbon. In this study, however, we used TS weight $\%$ for the bulk of discussion because that pyrite formation occurs not only in the water column but also in the sediments and hence $\%$ TS values are needed.

The sulfur isotopic composition $\left(\delta^{34} \mathrm{~S}\right)$ showed low values especially in Unit $1 / 6$. The $\delta^{34} \mathrm{~S}$ ranged from -40.9 to $-23.6 \%$ in Unit 3, from -44.9 to $-26.9 \%$, and from -58.5 to $-40.3 \%$ in Unit $1 / 6$. These low isotopic values mean that the abundant sulfur in the ACEX sediments was derived by the microbial reduction from sea water sulfate ion.
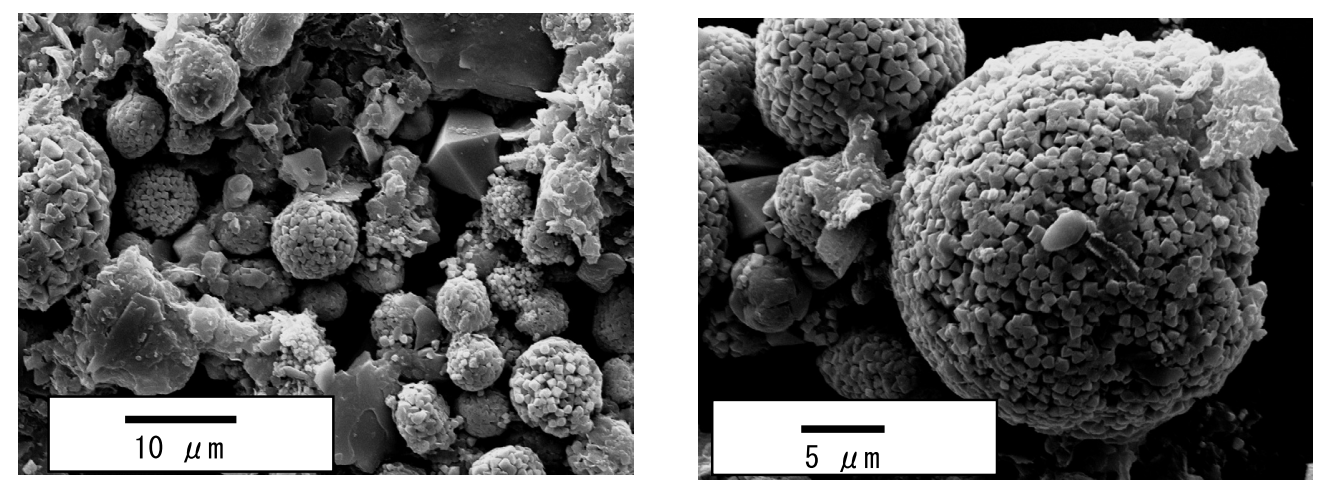

Fig. 5. Scanning electron photomicrographs of framboidal pyrites in 302-2A-50X-2, 36-38 belonging to Unit 1/6. 


\section{Discussion}

\section{1. Abundant sea water mass and its continuous supply into the Arctic Ocean}

The significantly high TS contents strongly suggests that significant sea water masses continuously existed in the Arctic Ocean throughout all the units investigated. Although the results of the previous microfossil analyses suggested that the middle Eocene Arctic Ocean had been fresh to brackish water environments (Backman et al., 2006; Onodera et al, in press), the low salinity condition must have been restricted within the upper water column, especially in the euphotic layer. This is because that most of the microfossils encountered were either phytoplankton or zooplankton with symbiotic organisms, which required light energy. The entering sea water from the outside of the basin sank to the deep depth of the basin, resulting in the formation of stratification due to the difference in salinity between the surface and deep water masses.

The $\%$ TS remarkably increased from Unit 2 to Unit 1/6, indicating further addition of sea water masses to the Arctic Ocean. This is coherent with the improved surface water exchange between the Arctic and the outside ocean suggested by the siliceous plankton assemblages (Onodera et al., in press). The sharp change of TS from Lithologic Unit 2 to Unit 1/6 probably suggests the increase of sea water supply was abrupt. In addition to the shift of TS, the extremely high TS value itself is remarkable. The sulfur contents of the middle Eocene Arctic Ocean is much higher than that of modern sediments under anoxic condition such as the Black Sea (e.g., Berner, 1984). This indicates the unique environments of the Arctic Ocean in accumulating sulfur distinctively different from the modern counterpart.

\section{2. Depositional environment reconstructed from TOC-TS plots}

The TS results are plotted against TOC (Fig. 6). The relationship between TOC and TS can be used to decipher the depositional environment. According to Berner and Raiswell (1983), under a normal marine condition where bottom water is well oxygenated the pyrite formation takes place only below the sediment-water interface and \%TOC-\%TS regression line tends to be on the line of $\mathrm{TS}=0.36 * \mathrm{TOC}$. By contrast, under freshwater environment much less pyrite is formed than in marine environment because of much lower concentrations of dissolved sulfate in freshwaters. Under euxinic conditions such as in the modern Black Sea, the bottom water contains dissolved $\mathrm{H}_{2} \mathrm{~S}$ and hence bacterial sulfate reduction occurs both in the water column and sediments resulting excess pyrite formation. The \%TOC-\%TS plots of euxinic conditions are indicated above the normal marine line. The plotted ACEX data are found in the domain of the euxinic condition in the diagram. Moreover, there are positive correlations between TS and TOC in Unit 3 and Unit 2 (Fig. 7). The regression lines represent $\mathrm{TS}=1.6 * \mathrm{TOC}+2.3(\mathrm{r}=0.71 ; \mathrm{n}=15)$ in Unit 3 , and $\mathrm{TS}=0.8 * \mathrm{TOC}+2.1$ $(\mathrm{r}=0.65 ; \mathrm{n}=92)$ in Unit 2 , respectively. Both regression lines have positive $\mathrm{S}$ intercepts. Leventhal (1983) suggested that the presence of a positive intercept is due to microbial sulfate reduction followed by pyrite formation in anoxic water column containing dissolved $\mathrm{H}_{2} \mathrm{~S}$. Thus the bottom water of the Arctic Ocean was anoxic and it contained dissolved $\mathrm{H}_{2} \mathrm{~S}$. The euxinic condition of the middle Eocene Arctic Ocean was also suggested by Stein et al. (2006). Several conditions caused the euxinic condition in the Arctic Ocean. The stratified water mass of the basin limited the oxygen supply from the surface to the deep water. In addition, the Arctic Ocean's semi-closed and isolated geography in the early and middle Eocene (Akhmetiev and Beniamovsk, 2004) restricted the circulation of the bottom water preventing the oxygen supply. The high productivity of the surface water (see below) also helped the development of the anoxic condition because oxidation of the organic matter in the water column consumed dissolved oxygen.

In Unit 1/6 a greater amount of excess sulfur relative to organic carbon was deposited than in Unit 2, suggesting a significant euxinic condition in this period. It also should be noted that the plotted pattern of this unit does not belong to the previously known patterns. This also implies the 


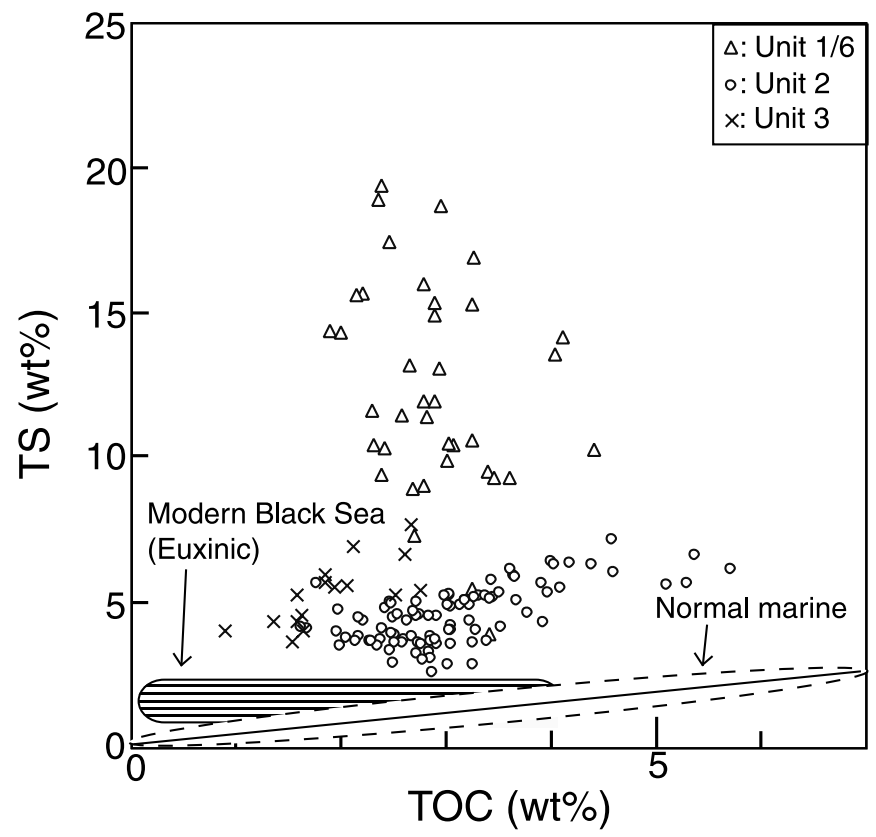

Fig. 6. Plots of TOC (wt\%) vs TS (wt \%) of the ACEX sediments. The domain enclosed by dashed lines represents normal marine sediments and the domain with horizontal lines represents modern euxinic Black Sea sediments (adapted from Berner and Raiswell, 1983).

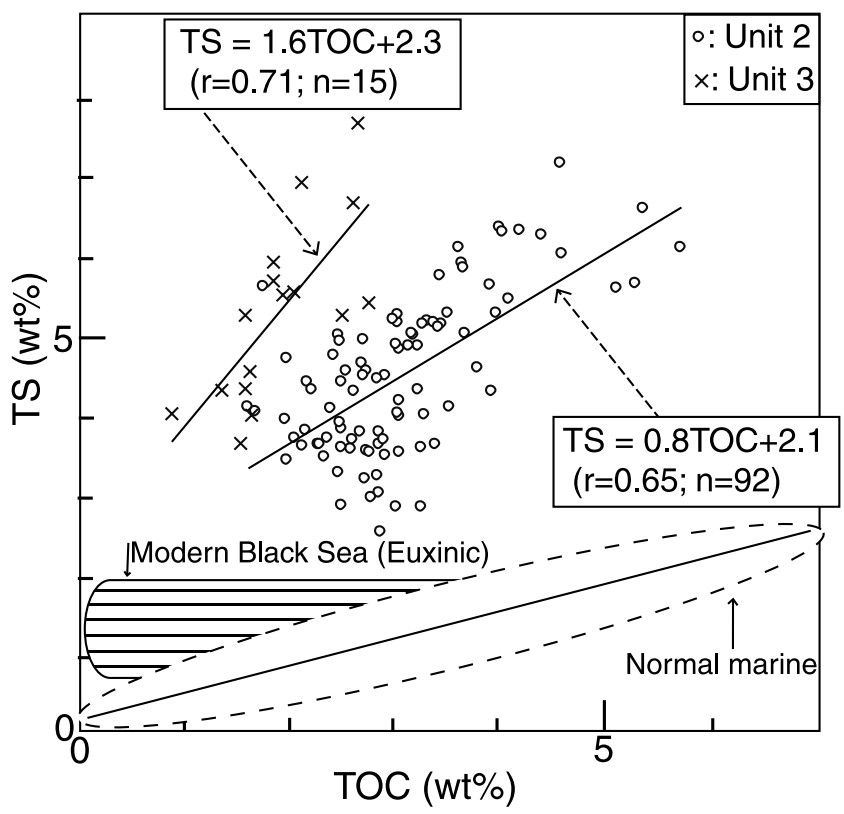

Fig. 7. Regression lines for the TOC vs TS data plot data from Units 2 and 3, respectively. The domains for normal marine and the modern euxinic Black Sea are adapted from Berner and Raiswell (1983). 
characteristic environment of the Arctic Ocean in this period which cannot be compared with the modern analogues.

\section{3. Paleo productivity of the middle Eocene Arctic Ocean}

The biogenic opal and TOC in the sediments are thought to represent paleoproductivity in the surface waters of the ocean (Leinen et al., 1986; Sarnthein et al., 1988). The source of organic matter, whether marine or terrestrial, however, must be characterized prior to the detailed discussion on marine paleoceanography. Organic matter type can be represented by TOC/N $(\mathrm{C} / \mathrm{N})$ ratios, which can illustrate the sources. Marine organic matter usually represents $\mathrm{C} / \mathrm{N}$ ratios ranging from 4 to 10 (Meyers, 1994) and the $\mathrm{C} / \mathrm{N}$ ratios of terrestrial plants represent greater than 20 (Hedges et al., 1986). The $\mathrm{C} / \mathrm{N}$ ratios of the ACEX samples were generally ranging from 10 to 20 with a mean value of 17.5 in all of the lithological units investigated. These values are rather high for marine organic matter. One explanation for the high $\mathrm{C} / \mathrm{N}$ ratios is that organic matter was composed of a mixture of marine and terrestrial origin. Taking into account the proximity of the past location of the Lomonosov Ridge to the Barrents/Kara Sea margin, the influence of terrestrial organic matter is reasonable. Furthermore, there is a possibility that microbial denitrification in anoxic conditions caused the preferential loss of organic nitrogen, resulting an increase of the $\mathrm{C} / \mathrm{N}$ ratios. The middle Eocene Arctic Ocean was significantly anoxic as discussed later. Therefore, if the high $\mathrm{C} / \mathrm{N}$ ratios were indeed caused by microbial denitrification, the organic matter in ACEX samples could have been mainly produced by marine plankton.

In order to reconstruct paleoproductivity without dilution effect by other parameters, biogenic opal AR and TOC AR were calculated. Biogenic opal contents were very high (mean value: 54.3\%) in the upper part of Unit 3 (ca. 54-49 Ma), although siliceous microfossils were absent judging from microscopic observations. The interval contains opal-CT which is comprised of cristobalite and tridymite (Backman et al., 2006), and opal-CT is converted from opal-A (siliceous microfossils) in early diagenesis (e.g., von Rad et al., 1977). Therefore, we interpret that siliceous microfossils were initially abundant in these intervals and the biogenic opal contents of Unit 3 must be reflecting the past biological productivity.

The biogenic opal value of the lowermost section (ca. $54 \mathrm{Ma}$ ) probably represents the initiation of opal accumulation on the Lomonosov Ridge because that biogenic opal is not present below this horizon (Backman et al., 2006). Biogenic opal AR was continuously high in Unit 3, indicating high biological productivity. In the lowermost part of Unit 2 after 49Ma, the biogenic opal AR decreased to nearly one half of the values shown in Unit 3. This probably reflected the Azolla event (Brinkhuis et al., 2006). During this period sea water mass decreased as discussed above and the salinity in the euphotic layer became low. Such an environmental change was unfavorable for siliceous biota, resulting in decrease of the productivity of siliceous biota. Soon after the Azolla event (48.6 Ma), biogenic opal AR recovered to the level as high as in Unit 3 and maintained for the remainders of Unit 2 with rather minor fluctuation until 45.4Ma (lithological unit boundary between Units 2 and 1/6). Thus the high productivity of the Arctic Ocean had been sustained for 9 Myrs from 54 Ma to 45.4 Ma. Such a steady productivity was brought about by the estuarine type circulation (Moran et al., 2006; Onodera et al., in press). The estuarine type circulation is comprised of the outflow of the surface freshwater or low salinity water and the inflow of the deep salty water which consequently received rich nutrients in the semi-closed basin (Berger, 1970). The nutrient rich deep water is convected up into the surface layer to mix up with the freshwater, part of which flows out of the basin, leading to a high productivity regime. Abundant deposits of the biogenic opal and organic matter are the characteristic sedimentation pattern of this circulation type. In the middle Eocene Arctic, the spill out of the freshwater is suggested by the freshwater fern Azolla (Brinkhuis et al., 2006). In addition the sufficient sea water supply from the outer ocean over a sill or sills is 
suggested by the sulfur isotopic composition analyses. The sea water must have sunk to the deep basin of the Arctic because of high density relative to the freshwater overlying. The sea water entering the Arctic probably have had high nutrients such as dissolved silicon because that the Paleocene and early Eocene active volcanism in the North Atlantic presumably supplied much of the source material for the dissolved silicon to the deep water of the North Atlantic Ocean (Williams, 1986). The high productivity of the Eocene Arctic Ocean was probably brought about by this high nutrient water inflow with the estuarine circulation.

The Eocene represents one of the global maxima for silica accumulation peaks in the Cenozoic sediments (McGowran, 1989) as well as what we have witnessed in the Arctic Ocean. The peak is recorded approximately from 49 to $45 \mathrm{Ma}$ and the biosiliceous sediments are referred to as Horizon $\mathrm{A}^{\mathrm{C}}$ in seismic reflection data. The horizon is found in the North Atlantic and the equatorial Pacific region (Racki and Cordey, 2000). The whole duration for Unit 2, which contains abundant biogenic opal, closely corresponds with the period represented by Horizon $\mathrm{A}^{\mathrm{C}}$. However, when opal-CT interval of the upper part of Unit 3 is taken as paleoproductivity, the timing of increased biogenic opal accumulation of the Arctic Ocean preceded the Horizon $\mathrm{A}^{\mathrm{C}}$ about $5 \mathrm{Myr}$ earlier. This supports the transition of biogenic opal deposition from the Arctic to the North Atlantic (Kitchell and Clark, 1982).

From Unit 2 to Unit 1/6, the biogenic opal AR decreased abruptly, indicating the decrease in the productivity of siliceous biota. The beginning of the surface sea water inflow from the North Atlantic (Onodera et al., in press) might have resulted in the low productivity because that the entry of the nutrient poor surface water could have contributed to the dilution of the nutrients in the surface layer of the Arctic basin. However, TOC AR increased in this unit relative to the unit below on the contrary. The reason for this discrepancy is the additional input of terrestrial organic matter in Unit 1/6. Dominance of terrestrial organic matter in Unit 1/6 was suggested by Stein et al. (2006). The terrestrial matter was probably transported by sea-ice or iceberg, which was suggested by the IRD occurrence in this unit (St. John, 2007). Furthermore, Unit 1/6 contains significantly higher amount of lithogenic material than in Unit 2, which made the distinction between the two units. Thus, the increased level of terrestrial matter input in Unit $1 / 6$ relative to the section below is apparent.

\section{4. Sulfur isotopic composition}

The $\delta^{34} \mathrm{~S}$ in Unit 3 and Unit 2 show constantly low values with a mean value of $-37 \%$. This result indicates an open system bacterial sulfate reduction where diffusion and exchange of sulfate is possible near the sediment-water interface or between the anoxic water column and the upper oxygenated layer. The continuous existence and supply of sea water into the Arctic is also suggested by the sulfur isotopic composition. The presence of freshwater in the Arctic was at the maximum extent at about $49 \mathrm{Ma}(\sim 300 \mathrm{mbsf})$ as the free-floating freshwater fern Azolla occurred abundantly (Brinkhuis et al., 2006). The \%TS shows about $5 \%$ at around this horizon and hence the Arctic Ocean did not become a freshwater basin entirely. However, this interval represents higher $\delta^{34} \mathrm{~S}$ values of $-26.9 \%$ than that of the upper and lower sequences $(-37 \%$ to $-38 \%$ ). This indicates that the Arctic Ocean became semi-closed system of bacterial sulfate reduction in which the exchange of sulfate was limited. This was because that the volume of sea water mass decreased in this period and the $\delta^{34} \mathrm{~S}$ of pyrite shifted to that of the source sea water $\mathrm{SO}_{4}{ }^{2-}$.

In Lithological Unit $1 / 6$, the $\delta^{34} \mathrm{~S}$ became extremely low which might mean the Arctic Ocean became more open to sulfate. This trend matches with the improved water connection between the Arctic and the outside basins such as the paleo-Atlantic which is suggested by the TS abundance and siliceous microfossiles (Onodera et al., in press). However, the extremely lower isotopic values than the usual isotopic values under normal marine sediments were observed, ranging from $-20 \%$ to 
$-40 \%$. The similar isotopic shift toward lower values was also observed in the carbon isotope analyses (Stein et al., 2006). These isotopic trends might represent the unique environment of the middle Eocene Arctic Ocean which is not observed in the modern world.

\section{Conclusions}

The geochemical analyses of ACEX sediments indicated the sufficient deep sea water presence in the middle Eocene Arctic where the low salinity environment was suggested by planktonic parameters. Probably the stratified water masses contributed to preventing the oxygen supply to the bottom layer leading to the significant anoxic condition. The influence of sea water from the outside basin appeared stronger from Unit 2 to Unit $1 / 6$ with the lithology change from Unit 2 to Unit $1 / 6$, which is coherent with the signals by siliceous plankton assemblages. The middle Eocene is thought to be the period in which the Arctic Ocean became more open to the outside basin. On the other hand, the abundant TS, the relationship between TOC and TS, and the extremely low sulfur isotopic values show unique patterns which cannot be simply compared with the other modern oceans. These results suggest that the middle Eocene Arctic Ocean hosted characteristically unique environments on the geochemical aspect.

\section{Acknowledgements}

We thank the co-chief scientists Prof. Jan Backman and Dr. Kate Moran, who materialized the ACEX, and the ACEX scientists as well as the captains and crew of the IODP Expedition 302 who assisted us in various phases of this work. We are grateful to Prof. Hitoshi Chiba of Okayama University for assistance in the sulfur isotope analyses. This research was partially supported by JSPS B Project No. 17310009 to KT.

\section{References}

Akhmetiev, M.A., and Beniamovski, V.N. (2004) Paleocene and Eocene of Western Eurasia (Russian sector) - stratigraphy, palaeogeography, climate. Neues Jahrbuch für Geologie und Paläontologie, Abhandlungen, 234, 137-181.

Backman, J., Moran, K., McInroy, D.B., Mayer, L.A., and the Expedition 302 Scientists. (2006) Proc. IODP, 302: College Station TX (Integrated Ocean Drilling Program Management International, Inc.). doi:10.2204/iodp.proc.302

Bak, F., and Cypionka, H. (1987) A novel type of energy metabolism involving fermentation of inorganic sulphur compounds. Nature, 326, 891-892.

Berger, W.H. (1970) Biogenous deep-sea sediments: fractionation by deep-sea circulation. Geol. Soc. Amer. Bull, 81, 1385-1402.

Berner, R.A. (1970) Sedimentary pyrite formation. American Journal of Science, 268, 1-23.

Berner, R.A., and Raiswell, R. (1983) Burial of organic carbon and pyrite sulfur in sediments over Phanerozoic time: a new theory. Geochemica et Cosmochimica Acta, 47, 855-862.

Berner, R.A. (1984) Sedimentary pyrite formation: An update. Geochemica et Cosmochimica Acta, 48, 605-615.

Böttcher, M.E., Smock, A., and Cypionka, H. (1998) Sulfur isotope fractionation during experimental precipitation of iron(II) and manganese(II) sulfide at room temperature. Chemical Geology, 146, 127-134. 
Brinkhuis, H., Schouten, S., Collinson, M.E., Sluijs, A., Sinninghe Damsté, J.S., Dickens, G.R., Huber, M., Cronin, T.M., Onodera, J., Takahashi, K., Bujak, J.P., Stein, R., van der Burgh1, J., Eldrett, J.S., Harding, I.C., Lotter, A.F., Sangiorgi, F., van Konijnenburg-van Cittert, H., de Leeuw, J.W., Matthiessen, J., Backman, J., Moran, K., and the Expedition Scientists. (2006) Episodic fresh surface waters in the Eocene Arctic Ocean. Nature, 441, 606-609.

Bukry, D. (1984) Paleogene paleoceanography of the Arctic Ocean is constrained by the middle or late Eocene age of the USGS core Fl-422; evidence from silicoflagellates. Geology, 12, 199-201.

Chambers, L.A., and Trudinger, P.A. (1979) Thiosulfate formation and associated isotope effects during sulfite reduction by clostridium-pasteurianum. Canadian journal of microbiology, 25, 719-721.

Dell'agnese, D.J., and Clark, D.L. (1994) Siliceous microfossils from the warm late Cretaceous and early Cenozoic Arctic Ocean. Journal of Paleontology, 68 (1), 31-47.

Hedges, J.I., Clark, W.A., Quay, P.D., Richey, J.E., Devol, A.H., and Santos, U.D. (1986) Composition and fluxes of particulate organic material in the Amazon River. Limnology Oceanography, 31, 717-738.

Holland, M.M., Bitz, C.M., Eby, M., and Weaver, A.J. (2001) The roll of ice-ocean interaction in the variability of the North Atlantic thermohaline circulation. Journal of Climate, 14, 656-675.

Jørgensen, B.B. and Fenchel, T. (1974) The sulfur cycle of a marine sediment model system. Marine Biology, 24, 189-201.

Jørgensen, B.B., (1990) A thiosulfate shunt in the sulfur cycle of marine sediments. Science, 239, 152-154.

Kajiwara, T. (1989) The potential contribution of biologically-controlled sulfur sources to ore genesis. Ann. Rep., Inst. Geosci., Univ. Tsukuba, 15, 96-101.

Kaplan, I.R., and Rittenberg, S.C. (1964) Microbiological fractionation of sulphur isotope. Journal of General Microbiology, 34, 195-212.

Kitchell, J.A., and Clark, D.L. (1982) Late Cretaceous-Paleogene paleogeography and palercirculation: Evidence of north polar upwelling. Palaeogeography, Palaeoclimatology, Palaeoecology, 40, 135-163.

Krämer, M., and Cypionka, H. (1989) Sulfate formation via ATP sulfurylase in thiosulfate- and sulfide-disproportionating bacteria. Archives of microbiology, 151. 232-237.

Leinen, M., Cwienk, D., Heath, G.R., Biscaye, P.E., Kolla, V., Thiede, J., and Dauphin, J.P. (1986) Distribution of biogenic silica and quartz in recent deep-sea sediments. Geology, 14, 199-203.

Leventhal, J.S. (1983) An interpretation of carbon and sulfur relationship in Black Sea sediments as indicators of environments of deposition. Geochemisca et Cosmohimica Acta, 47, 133-137.

McGowran, B. (1989) Silica burp in the Eocene ocean. Geology, 17, 857-860.

Meyers, P.A. (1994) Preservation of elemental and isotopic source identification of sedimentary organic matter. Chemical Geology, 114, 289-302.

Moran, K., Backman, J., Brinkhuis, H., Clemens, S.C., Cronin, T., Dickens, G.R., Eynaud, F., Gattacceca, J., Jakobsson, M., Jordan, R.W., Kaminski, M., King, J., Koc, N., Krylov, A., Martinez, N., Matthiessen, J., McInroy, D., Moore, T.C., Onodera, J., O’Regan, M., Pälike, H., Rea, B., Rio, D., Sakamoto, T., Smith, D.C., Stein, R., St. John, K., Suto, I., Suzuki, N., Takahashi, K., Watanabe, M., Yamamoto, M., Farrell, J., Frank, M., Kubik, P., Jokat, W., and Y. Kristoffersen. (2006) The Cenozoic palaeoenvironment of the Arctic Ocean. Nature, 441, 601605.

Mortlock, R.A., and Froelich, P.N. (1989) A simple method for the rapid determination of biogenic opal in pelagic sediments. Deep-Sea Research, 36, 1415-1426.

Moses, C.O., Nordstrom, D.K., Herman, J.S., and Mills, A.L. (1987) Aqueous pyrite oxidation by dissolved-oxygen and by ferric iron. Geochemica et Cosmochimica Acta, 51. 1561-1571. 
Müller, P.J., and Suess, E. (1979) Productivity, sedimentation rate, and sedimentary organic matter in the ocean. I. Organic carbon preservation. Deep-Sea Research, 26A, 1347-1362.

Nakai, N., Ohta, T., Fujisawa, H., and Yoshida, M. (1982) Paleoclimatic and sea-level changes deduced from organic carbon isotope ratio, $\mathrm{C} / \mathrm{N}$ ratios and pyrite contents of cored sediments from Nagoya Harbor, Japan. The Quaternary Research, 21, 169-177.

Ohmoto, H. and Rye, R.O. (1979) Isotope of sulfur and carbon, in Geochemistry of Hydrothermal Ore Deposits (2nd edition), edited by H.L. Barnes, 509-567, John Wiley and Sons, New York.

Onodera, J., Takahashi, K., and Jordan, R.W. The Eocene silicoflagellate and ebridian paleoceanography in the central Arctic Ocean. Paleoceanography (in press).

Racki, G., and Cordey, F. (2000) Radiolarian palaeoecology and radioolarites: is the present the key to the past? Earth-Science Reviews, 52, 83-120.

Sarnthein, M., Winn, K., Duplessy, J.-C., and Fortugne, M. R., (1988) Global carbon variations of surface ocean productivity in low and mid latitudes: influence on $\mathrm{CO}_{2}$ reservoirs of the deep ocean and atomosphere during the last 21,000 years. Paleoceanogr., 3(3), 361-399.

Stein, R., Boucsein, B., and Meyer, H. (2006) Anoxia and high primary production in the Paleogene central Arctic Ocean: First detailed records from Lomonosov Ridge. Geophysical Research Letters, 33. L18606, doi:10.1029/2006GL026776.

St. John, K.E. (2007) Neogene and Eocene Ice-Rafting in the Central Arctic. AGU 2007 Fall Meeting, San Francisco, USA. 10-14 December 2007, Eos Trans. AGU, Fall Meet. Suppl., Abstract PP43D-03.

Vogt, P.R., Taylor, P.T., Kovacs, L.C., and Johnson, G.J. (1979) Detailed aeromagnetic investigation of the Arctic Basin. Journal of Geophysical research, 84, 1071-1089.

von Rad, U., Riech, V., and Rösch, H. (1977) Silica diagenesis in continental margin seiments off northwest Africa. In Lancelot, Y., Seibold, E., et al., (1977) Initial Reports of the Deep Sea Drilling Project, 41: Washington (U.S. Government Printing Office), pp. 879-906.

Williams, C.A. (1986) An oceanwide view of Palaeogene plate tectonic events. Palaeogeography, Palaeoclimatology, Palaeoecology, 57, 3-25.

Zachos, J., Pagani, M., Sloan, L., Thomas, E., and Billups, K. (2001) Trends, rhythms, and aberrations in global climate 65 Ma to present. Science, 292, 686-693. 